\title{
LITTLEWOOD'S DIOPHANTINE APPROXIMATION PROBLEM FOR SERIES
}

T. W. CUSICK

1. The well-known Diophantine approximation problem of Littlewood is whether for each pair of real numbers $\theta, \phi$ and each $\epsilon>0$ there exists a positive integer $n$ such that

$$
n\|n \theta\|\|n \phi\|<\epsilon,
$$

where $\|\alpha\|$ is the absolute value of the difference between $\alpha$ and the nearest in teger.

Let $K$ be a given field of characteristic zero. If we consider the field $K\{t\}$ of formal power series

$$
\gamma=a_{m} t^{m}+a_{m-1} t^{m-1}+\cdots
$$

with coefficients in $K$ and define a valuation on $K\{t\}$ by

$$
|\gamma|=e^{m}
$$

where $a_{m} \neq 0$ and $m$ may be positive, negative, or zero, then there is an analogue for $K\{t\}$ of Littlewood's problem for the real numbers. The field $K\{t\}$ corresponds to the real numbers, the ring $K[t]$ of polynomials with coefficients in $K$ corresponds to the rational integers, and we define the analogue of the distance to the nearest integer function by $\|\gamma\|=e^{-h}$, where $a_{-1}=a_{-2}=\cdots=a_{-h+1}=0$, $a_{-h} \neq 0$.

The analogue of Littlewood's problem was solved by Davenport and Lewis in [2]. They proved that there exist $\theta, \phi$ in $K\{t\}$ such that

$$
|p|\|p \theta\|\|p \phi\| \geqq e^{-2}
$$

for all $p \neq 0$ in $K[t]$. Explicit examples of $\theta$ and $\phi$ satisfying (1) with $e^{-5}$ in place of $e^{-2}$ were given in [1], with generalizations relating to products with an arbitrary number of factors. We prove the following result, which gives still more information about the analogue of Littlewood's problem and extensions of it:

THEOREM 1. Given an integer $q \geqq 2$, let $f(t)$ be an arbitrary polynomial of degree at least $q$ in $K[t]$. Then there exist continuum-many $\theta$ in $K\{t\}$ such that

$$
|p|\left(\prod_{i=1}^{q-1}\left\|p \theta^{i}\right\|\right)\|p f(\theta)\| \geqq e^{-q}
$$

Received by the editors October 4, 1966. 
far all $p \neq 0$ in $K[t]$.

If we put $f(t)=t^{q}$, then Theorem 1 becomes

THEOREM 2. Given an integer $q \geqq 2$, there exist continuum-many $\theta$ in $K\{t\}$ such that

$$
|p| \prod_{i=1}^{q}\left\|p \theta^{i}\right\| \geqq e^{-q}
$$

for all $p \neq 0$ in $K[t]$.

The case $q=2$ of Theorem 2 shows that we can take $\phi=\theta^{2}$ and still obtain the inequality (1) for all $p \neq 0$ in $K[t]$.

It is easily seen that the constant $e^{-q}$ in Theorems 1 and 2 cannot be improved. In fact the product in (2) or (3) is $\leqq e^{-q}$ when $p=1$, and indeed for infinitely many $p$ by the analogue for power series of Minkowski's theorem on linear forms.

We remark also that the condition that $K$ has characteristic zero can be somewhat relaxed; a more detailed examination of the proofs shows that it suffices if $K$ contains infinitely many elements and its characteristic, if it is nonzero, is sufficiently large.

I am grateful to Dr. A. Baker for helpful discussions.

2. It is simplest to prove the case $q=2$ of Theorem 2 first, for the other cases follow from a generalization of that proof. Theorem 1 is obtained by extending the method of proof of Theorem 2 .

We shall prove the existence of

$$
\theta=a_{0}+a_{1} t^{-1}+a_{2} t^{-2}+\cdots
$$

such that (3) is satisfied for $q=2$ and all $p \neq 0$ in $K[t]$ by successively choosing the $a_{i}$ 's. Let

$$
\theta^{2}=b_{0}+b_{1} t^{-1}+b_{2} t^{-2}+\cdots,
$$

so that

$$
b_{i}=a_{0} a_{i}+a_{1} a_{i-1}+\cdots+a_{i} a_{0} .
$$

In what follows we regard each $a_{i}$ as a variable until it has been asserted that some fixed value in $K$ has been assigned to it. The use of the symbol $a_{i}$ both for a variable and for a value taken by that variable should, as usual, cause no confusion.

As pointed out in [2], it is easily verified that a necessary and sufficient condition that (3) hold for $q=2$ and for all $p \neq 0$ in $K[t]$ is that the determinants 


$$
\delta_{(i, j)}=\operatorname{det}\left|\begin{array}{llll}
a_{1} & a_{2} & \cdots & a_{i+j} \\
a_{2} & a_{3} & \cdots & a_{i+j+1} \\
\dot{b} & \dot{\cdot} & & \dot{\cdot} \\
\dot{a_{i}} & \dot{a}_{i+1} & \cdots & a_{2 i+j-1} \\
b_{1} & b_{2} & \cdots & b_{i+j} \\
b_{2} & b_{3} & \cdots & b_{i+j+1} \\
\cdot & \cdot & & \cdot \\
\dot{b_{j}} & \dot{b}_{j+1} & \cdots & \dot{b}_{i+2 j-1}
\end{array}\right|
$$

be nonzero for each pair $i, j$ with $i \geqq 0, j \geqq 0, i+j \geqq 1$.

In the proof we make use of the following evident relations, true for each pair $i, j$ specified above:

$$
\begin{aligned}
& \delta_{(i, j)}=(-1)^{j} a_{2 i+j-1} \delta_{(i-1, j)}+f_{i, j}\left(a_{0}, a_{1}, \cdots, a_{2 i+j-2}\right) \quad(i>j), \\
& \text { (6) } \delta_{(i, j)}=2 a_{0} a_{i+2 j-1} \delta_{(i, j-1)}+f_{i, j}\left(a_{0}, a_{1}, \cdots, a_{i+2 j-2}\right)
\end{aligned}
$$

Here the $f_{i, j}$ are polynomials in the specified $a_{i}$ 's only.

Consider the variables $a_{k}$ occurring in the matrix of $\delta_{(i, j)}$ and call the variable with largest suffix $k$ the leading variable of $\delta_{(i, j)}$. We notice that all of the $f_{i, j}$ are independent of the leading variables of their respective $\delta$ 's.

We begin our selection of the $a_{i}$ 's by taking $a_{0}$ and $a_{1}$ nonzero but otherwise arbitrary in $K$. We show that the remaining $a_{i}$ 's can be chosen appropriately by an inductive argument which depends on the fact that the coefficient of the highest power of $a_{m}$, viz. $a_{m}^{3 m}$, in $\delta_{(m, m)}$ is not zero. In fact this coefficient is +1 , as is clear from the form of the matrix for $\delta_{(m, m)}$ if we notice that the only $b_{i}, 0 \leqq i \leqq 3 m-1$, which contains $a_{m}$ to a power higher than the first is $b_{2 m}$. Since $\delta_{(m, m)}$ contains a term in $a_{m}$ alone with nonzero coefficient,

$$
\delta_{(i, i)} \not \equiv 0 \text { as a polynomial in } a_{i} \text { for each } i=1,2,3, \cdots \text {, }
$$

irrespective of the values assigned to the other variables in $\delta_{i, i}$.

Since $a_{0} a_{1} \neq 0$, also $\delta_{(1,0)}=a_{1} \neq 0, \delta_{(0,1)}=2 a_{0} a_{1} \neq 0$, and $\delta_{(1,1)}=a_{1}^{3} \neq 0$. We next fix $a_{2}$ in $K$ in such a way that $\delta_{(2,2)} \not \equiv 0$ in its remaining variables $a_{3}, a_{4}, a_{5}$; this is possible by the case $i=2$ of (7).

Now suppose that for some integer $n \geqq 2$ we have chosen $a_{0}, a_{1}, \cdots$, $a_{n}$ in $K$ in such a way that

1. Each $\delta_{(i, i)}$ whose leading variable has suffix $\leqq n$ (i.e., each $\delta_{(i, i)}$ such that $3 i-1 \leqq n)$ is not zero.

2. Each $\delta_{(i, i)}, i \leqq n$, whose leading variable has suffix $>n$ is not identically zero in its remaining variables $a_{n+1}, a_{n+2}, \cdots, a_{3 i-1}$. 
3. Each $\delta_{(i, j)}, i \neq j$, whose leading variable has suffix $\leqq n$ is not zero.

Our remarks above have established conditions 1,2 and 3 for $n=2$, so we assume 1, 2 and 3 for $n=k \geqq 2$ and show that we can choose $a_{k+1}$ in $K$ in such a way that conditions 1,2 and 3 hold for $n=k+1$.

Condition 1 for $n=k+1$ follows at once from the same statement for $n=k$ unless $k+1=3 I-1$ for some $I$. In the latter case we must choose $a_{k+1}$ so that $\delta_{(I, I)} \neq 0$ (this is possible by condition 2 with $n=k$ ), i.e. our choice of $a_{k+1}$ must avoid a finite number of values.

Condition 2 for $n=k+1$ follows from the same statement for $n=k$ and the fact that, by the case $i=k+1$ of (7), we can choose $a_{k+1}$ in such a way that $\delta_{(k+1, k+1)} \neq 0$ in its remaining variables $a_{k+2}, a_{k+3}, \cdots$, $a_{3 k+2}$. In order to do this our choice of $a_{k+1}$ must avoid a finite number of values.

Condition 3 for $n=k+1$ can be satisfied by the same statement for $n=k$ and (5), (6). For there is only a finite number of $\delta_{(i, j)}, i \neq j$, whose leading variable is $a_{k+1}$, and in each of these, by the induction hypothesis and (5) or (6), $a_{k+1}$ appears linearly with a nonzero coefficient. Thus we need only avoid a finite number of values of $a_{k+1}$ in order to make each of these $\delta_{(i, j)}$ not zero.

Combining the above statements, we see that provided we avoid a finite number of values we can fix $a_{k+1}$ in $K$ in such a way that 1,2 and 3 are satisfied with $n=k+1$. Hence by induction we can choose $a_{0}, a_{1}, a_{2}, \ldots$ in $K$ so that 1,2 and 3 are satisfied for each $n=2,3$, $4, \cdots$.

This gives the case $q=2$ of Theorem 2 except for the statement that continuum-many $\theta$ of the required type exist; but, since in the above we have an infinite number of choices for each $a_{i}$, the desired statement follows.

3. The above method of proof is easily generalized to give Theorem 2 for the case of arbitrary $q$. Let $\left.\delta_{(i(1), i(2)}, \ldots, i(q)\right)$ be the natural generalization of $\delta_{(i, j)}$ in $(4)$, so $\left.\delta_{(i(1), i(2)}, \ldots, i(q)\right)$ is the determinant of a certain matrix whose elements are coefficients of $\theta, \theta^{2}, \cdots, \theta^{q}$.

We divide the $q$-tuple subscript $\delta$ 's into two classes. We put in class $A$ those $\delta$ 's whose $q$-tuple subscript has $i(k)=\max _{1 \leqq j \leqq q} i(j)$ for only one value of $k$. We put in class $B$ all other $\delta$ 's, i.e. those $\delta$ 's whose $q$-tuple subscript has at least two equal elements bigger than the remaining elements.

With the obvious generalization of the definition of leading variable, our induction hypothesis is:

1. Each $\delta$ in class $B$ whose leading variable has suffix $\leqq n$ is not zero. 
2. Each $\delta$ in class $B$ with $\max _{1 \leq j \leq q} i(j) \leqq n$ whose leading variable has suffix $>n$ is not identically zero in its remaining variables.

3 . Each $\delta$ in class $A$ whose leading variable has suffix $\leqq n$ is not zero.

For each $\delta$ in class $A$ we have an identity like (5) or (6). Given a $\delta$ in class $B$, let $m=\max _{1 \leq j \leq q} i(j)$. Then by considering the coefficient of the highest power of $a_{m}$ in this $\delta$, we see that if $a_{0}, a_{1}, \cdots, a_{m-1}$ have already been chosen so as to satisfy 1,2 and 3 above for $n=m-1$, then the given $\delta$ is not identically zero as a polynomial in $a_{m}$. Thus for each $\delta$ in class $B$ we obtain an analogue of statement (7).

Using the above remarks, we may proceed with the induction for the case of general $q>2$ as in the case $q=2$, but with some complications of notation. This completes the proof of Theorem 2 .

4. In proving Theorem 1 , we begin with the case $q=2$. Suppose

$$
f(t)=c_{k} t^{k}+c_{k-1} t^{k-1}+\cdots+c_{0}, \quad c_{k} \neq 0, k \geqq 2,
$$

and let

$$
f(\theta)=g(t)+b_{0}^{\prime}+b_{1}^{\prime} t^{-1}+b_{2}^{\prime} t^{-2}+\cdots,
$$

where $g(t)$ is a polynomial with zero constant term. Define $\delta_{(i, j)}^{\prime}$ to be $\delta_{(i, j)}$ with each $b_{i}$ replaced by $b_{i}^{\prime}$ in (4). Then a necessary and sufficient condition that (2) hold for $q=2$ and for all $p \neq 0$ in $K[t]$ is that the determinants $\delta_{(i, j)}^{\prime}$ be nonzero for each pair $i, j$ with $i \geqq 0, j \geqq 0$, $i+j \geqq 1$.

We note that $b_{i}^{\prime}$ depends on $a_{0}, a_{1}, \cdots, a_{i}$ and that the only $b_{i}^{\prime}$, $0 \leqq i \leqq 3 m-1$, which contain $a_{m}$ to a power higher than the first are those with $i \geqq 2 m$. Therefore (5), (6), and (7) are still true if $\delta_{(i, j)}$ is replaced by $\delta_{(2, j)}^{\prime}$ throughout. Thus the case $q=2$ of Theorem 1 may be proved inductively in the same way as the case $q=2$ of Theorem 2 . We need only notice that at the beginning of the induction we must choose $a_{0}$ in such a way that $a_{0}$ is not zero and the coefficient of $a_{1}^{2}$ in $b_{2}^{\prime}$ is not zero (whence a fortiori the coefficient of $a_{m}^{2}$ in $b_{2 m}^{\prime}$ is not zero for $m=1,2,3, \cdots)$.

The generalization of the proof of Theorem 1 for the case $q=2$ to the proof for general $q$ is carried out in the same way as the corresponding generalization for Theorem 2 .

\section{REFERENCES}

1. A. Baker, On an analogue of Littlewood's Diophantine approximation problem, Michigan Math. J. 11 (1964), 247-250.

2. H. Davenport and D. J. Lewis, An analogue of a problem of Littlewood, Michigan Math. J. 10 (1963), 157-160.

Churchill College, Cambridge, England 